\title{
Dynamics of Colloidal Dispersions via Lattice-Gas Models of an Incompressible Fluid
}

\author{
A.J.C. Ladd ${ }^{1}$ and D. Frenkel ${ }^{2}$ \\ ${ }^{1}$ Lawrence Livermore National Laboratory, Livermore, CA94550, USA \\ 2F.O.M. Institute for Atomic and Molecular Physics, \\ NL-1009 DB Amsterdam, The Netherlands
}

\begin{abstract}
Progress in applying lattice-gas models to the simulation of colloidal dispersions is described. A new set of collision rules for the four-dimensional face-centeredhypercubic lattice, which lead to an exactly isotropic viscosity and which can be implemented with a small table lookup, have been developed. However, they are only suitable for low Reynolds number flows. A new implementation of a constant-velocity boundary condition for lattice-gas models is described.
\end{abstract}

The dynamical properties of colloidal dispersions are complex and difficult to understand or predict quantitatively. Consequently it is valuable to carry out computer simulations of simplified systems, such as monodisperse suspensions of rigid, electrically neutral spheres, so as to be able to study, for instance, the competition between Brownian and hydrodynamic forces and their effect on the transport properties such as viscosity and diffusion. There are already a number of very clean experimental studies of monodisperse latex spheres in suspension [1,2] and computer simulations could effectively complement these studies. The primary problem in simulating a dispersion lies in solving the fluid equations, subject to the complex boundary conditions imposed by the solid particles, and then determining the resulting forces and torques on the particles. A latticegas model of the fluid phase contains, in a natural fashion, the essential physics of the suspending fluid, namely the dissipative hydrodynamic forces, arising from macroscopic fluid motion, and the fluctuating forces, arising from random collisions between solvent and solute particles, that give rise to Brownian motion.

A preliminary account of the application of lattice-gas models to solid-fluid suspensions was reported last year by Ladd et. al. [3]. The essence of the method was a stochastic implementation of a non-zero velocity boundary condition in the lattice-gas fluid in which the fluid velocity in the immediate vicinity of the boundary surface was set, on average, to the desired local value. However, this boundary condition did not take into account the input state and is thus rather ineffective in the case of strong velocity gradients in the fluid. In particular, for a stationary solid surface an exact stick boundary condition is only obtained in the absence of local velocity gradients. During the course of this workshop some discussion was directed towards the problem of a implementing a constant-velocity boundary condition more effectively, and it was considered essential that the rules at the boundary should reduce to the usual "bounce-back" rule for a stationary surface. A stochastic but local implementation of this new boundary condition will be described here. The basic idea is to apply a force density at the boundary surface so as to achieve the required local velocity [4]. Moreover, we no longer exclude fluid from the 
interior of the solid particle but instead decouple it, more or less, from the from the exterior fluid via the imposed force density. This decoupling is complete for a stationary solid object, leading to the usual "bounce-back" rule for the hydrodynamic stick boundary condition. The implementation of the boundary condition exploits an idea of Rem and Somers [5], which involves applying the boundary condition at the halfway point between the nodes. Thus the boundary surface lies, not at the nodes as in the usual implementations, but midway between them. Consequently a particle encountering a stationary boundary surface finds itself after one timestep at the same site but with a reversed velocity, rather than at the site on the other side of the boundary. In fact this allows for a clearer definition of the boundary surface, since particles are either on one side or the other when undergoing collisions with each other. Moreover, the collision rules at the nodes are the same for all sites; the boundary conditions are applied after propagating the particles for half a time step and are followed by a further propagation of half a time step.

To see how the boundary-condition rules are implemented, consider a link $i$ connecting two sites on either side of the boundary surface, so that the boundary condition is to be applied at the point halfway between these two sites. If the boundary is stationary, particles are reflected back to their original lattice sites, thus implementing the "bounceback" rule at the half-node positions. If the boundary is moving locally in the direction $i$, that is $\mathbf{v} \cdot \mathbf{c}_{\mathrm{i}}>0$ with $\mathbf{v}$ the local velocity of the boundary and $\mathbf{c}_{\mathrm{i}}$ the velocity of link $i$, then particles of velocity $\mathbf{c}_{\mathrm{i}}$ are sometimes allowed to penetrate the boundary surface, whereas particles with velocity $\mathbf{c}_{-\mathrm{i}}=-\mathbf{c}_{\mathrm{i}}$ are always reflected. The transmission probability $p$ required to get the local equilibrium distribution corresponding to a velocity $\mathbf{v}$ can be calculated easily. Since we are specifically interested in linear flows, we will consider the case with a mean occupancy of each link of 0.5 when the non-linear advection term drops out of the lattice-gas hydrodynamic equations; generalization to other densities is straightforward.

If the local fluid velocity at the border halfway between two nodes is $\mathbf{v}$, then it follows from the local equilibrium distribution function $\mathrm{f}_{\mathrm{i}}$ that

$$
\begin{aligned}
& f_{1}=\mathrm{f}_{\mathrm{i}} \overline{\mathrm{f}}_{-\mathrm{i}}=\frac{1}{4}+2 \frac{\mathbf{v} \cdot \mathbf{c}_{\mathrm{i}}}{\mathrm{c}_{\mathrm{i}}^{2}}+4 \frac{\left(\mathbf{v} \cdot \mathbf{c}_{\mathrm{i}}\right)^{2}}{\mathrm{c}_{\mathrm{i}}^{4}}+\cdots, \\
& f_{2}=\overline{\mathrm{f}}_{\mathrm{i}} \mathrm{f}_{-\mathrm{i}}=\frac{1}{4}-2 \frac{\mathbf{v} \cdot \mathbf{c}_{\mathrm{i}}}{\mathrm{c}_{\mathrm{i}}^{2}}+4 \frac{\left(\mathbf{v} \cdot \mathbf{c}_{\mathrm{i}}\right)^{2}}{\mathrm{c}_{\mathrm{i}}^{4}}+\cdots,
\end{aligned}
$$

where $\overline{\mathrm{f}}_{\mathrm{i}}=1-\mathrm{f}_{\mathrm{i}}$ is the probability that link $i$ is empty. Note that this expansion is only correct to order $\mathrm{v}^{3}$ at half occupancy; at other densities further quadratic terms in $\mathbf{v}$, involving the second-rank momentum tensor $\mathbf{c}_{\mathbf{i}} \mathbf{c}_{\mathrm{i}}$, are non zero. The boundary conditions are implemented by transitions between states $f_{1}$ and $f_{2}$ at the halfway point between the nodes; of course the other two states containing 0 and 2 particles cannot be changed if local mass conservation is to be maintained. Let us consider the case where $x=4 \mathbf{v} \cdot c_{i} / c_{i}{ }^{2}$ is greater than zero; the reverse case can be generated by permuting the $i$ and $-i$ directions. To apply the moving boundary condition, state $(\bar{i},-i)$ is always converted to state $(i,-\bar{i})$, whereas $(i,-\bar{i})$ is converted to $(\bar{i},-i)$ with a reduced probability $(1-p)$ and remains invariant with probability $p$. Thus 


$$
f_{1}^{\prime}=p f_{1}+f_{2} ; f_{2}^{\prime}=(1-p) f_{1} \text {, }
$$

and in order for a steady state to exist with a local velocity $\mathbf{v}$, we require that

$$
p=\frac{4 \mathrm{x}}{(1+\mathrm{x})^{2}},
$$

whereupon the distributions given in Eqs. (1) and (2) are stationary.

After applying the boundary collision rule to an arbitrary velocity distribution, the new one-particle densities are given by

$$
\mathrm{f}_{\mathrm{i}}^{\prime}=\mathrm{f}_{\mathrm{i}} \mathrm{f}_{-\mathrm{i}}+\overline{\mathrm{f}}_{\mathrm{i}} \mathrm{f}_{-\mathrm{i}}+p \mathrm{f}_{\mathrm{i}} \overline{\mathrm{f}}_{-\mathrm{i}}=\mathrm{f}_{-\mathrm{i}}+p \mathrm{f}_{\mathrm{i}} \overline{\mathrm{f}}_{-\mathrm{i}},
$$

and similarly

$$
\mathrm{f}_{-\mathrm{i}}^{\prime}=\mathrm{f}_{\mathrm{i}}-p \mathrm{f}_{\mathrm{i}} \overline{\mathrm{f}}_{-\mathrm{i}} \text {. }
$$

The contribution of link $i$ to the local velocity is just the average of the velocities before and after the application of the boundary collision rule,

$$
\mathbf{v}_{\mathrm{i}}^{\prime}=\frac{1}{2}\left(\mathrm{f}_{\mathrm{i}} \mathbf{c}_{\mathrm{i}}+\mathrm{f}_{-\mathrm{i}} \mathbf{c}_{-\mathrm{i}}+\mathrm{f}_{\mathrm{i}}^{\prime} \mathbf{c}_{\mathrm{i}}+\mathrm{f}_{-\mathrm{i}}^{\prime} \mathbf{c}_{-\mathrm{i}}\right)=p \mathrm{f}_{\mathrm{i}} \overline{\mathrm{f}}_{-\mathrm{i}} \mathbf{c}_{\mathrm{i}}
$$

since $\mathrm{f}_{\mathrm{i}}+\mathrm{f}_{-\mathrm{i}}=\mathrm{f}_{\mathrm{i}}{ }^{\prime}+\mathrm{f}_{\mathrm{i}}^{\prime}=1$, apart from small corrections of order $\mathrm{v}^{2}$. The new velocity $\mathbf{v}^{\prime}$ is obtained by averaging over the 12 link directions

$$
\mathbf{v}^{\prime}=\frac{1}{12} \sum_{\mathrm{i}=1}^{12} p \mathrm{f}_{\mathrm{i}} \overline{\mathrm{f}}_{-\mathrm{i}} \mathbf{c}_{\mathrm{i}}=\frac{1}{12} \sum_{\mathrm{i}=1}^{12} 4 \mathbf{v} \cdot \frac{\mathbf{c}_{\mathrm{i}} \mathbf{c}_{\mathrm{i}}}{\mathrm{c}_{\mathrm{i}}^{2}}+\cdots,
$$

and is equal to the required velocity $\mathbf{v}$ irrespective of the initial distribution, again apart from small corrections of order $\mathrm{v}^{3}$.

To model the fluid phase of a three-dimensional colloidal dispersion, we have implemented the four-dimensional face-centered-hypercubic lattice-gas model proposed by Frisch et. al. [6], which has a high enough symmetry for an isotropic shear viscosity. Three dimensional flows are simulated by projecting onto a simple-cubic lattice. This lattice-gas model has 24 velocities, corresponding to the four diagonals in each of the 6 planes of the lattice $w x, w y, w z, x y, x z, y z$, where $w$ indicates the fourth spatial dimension. To implement the collision rules for this model by table lookup requires $2^{24} \approx 16 \times 10^{6}$ entries which in turn requires a very large shared memory. We have designed an algorithm which involves taking collisions between subsets of 12 directions, which reduces the memory requirement to around $2^{12} \approx 4000$ entries. In addition, our algorithm is simple to implement and leads to a fairly small and exactly isotropic viscosity tensor. For instance, at $\mathrm{d}=0.5$ (half occupancy), the kinematic viscosity is about $0.1 l^{2} / \Delta \mathrm{t}$ where $l$ is the lattice spacing and $\Delta \mathrm{t}$ is the time step, and the mean-free path is less than one lattice spacing. This is more than adequate for low-Reynolds number flows that occur in colloidal dispersions or in flows through porous media but for high-Reynolds number flows the algorithm is much less effective. The parameter $\mathrm{Re}_{*}^{\max }$ measuring the effectiveness of the collision 
rules [6], is about 2, similar to Henon's isometric rules [7], but much less than the value 7.1 obtained with an optimized table lookup [8]. Our method is implemented as follows.

The six planes of the FCHC lattice are coupled together in two sets of three and within each set there are 2 indexes which occur twice and two indexes which occur once, for instance wx-wy-xz and wz-xy-yz. There are 5 other such couplings. Any state of the 24 bit model can be split into 12 bit substates and each substate can be reduced to a state of a generic sublattice such as wx-wy-xz by a few logical operations. After determining the outcome of the collision for both substates from a small table lookup, the final output state can be assembled by a reverse sequence of logical operations. However, permutation of the planes does not generate all the symmetry operations of the lattice, so isotropy of the viscosity will not be achieved for an arbitrary set of collision rules. Random selection of possible couplings of planes ensures symmetry with respect to permutation of suffixes, but full isotropy requires an equivalence between the diagonal and off-diagonal components of the viscosity tensor. In our collision rules this diagonal off-diagonal equivalence is enforced separately for all states with a given number of particles, thereby ensuring isotropy at all densities. Implementation of these collision rules and boundary conditions for three-dimensional colloidal dispersions is in progress.

Acknowledgments. We wish to acknowledge that discussions with several participants at the workshop, and in particular with Peter Rem (Shell Research, Amsterdam), were instrumental in developing the boundary conditions described here.

This work was supported by the U.S. Department of Energy and Lawrence Livermore National Laboratory under Contract No. W-7405-Eng-48.

The work of the FOM Institute is part of the scientific program of FOM and is supported by the 'Nederlandse Organisatie voor Wetenschappelijk Onderzoek' (NWO).

\section{References}

[1] F. Gadala-Maria and A. Acrivos, J. Rheol., 24, 799 (1980).

[2] G.L. Paul and P.N. Pusey, J. Phys. A, 14, 3301 (1981).

[3] A.J.C. Ladd, M.E. Colvin and D. Frenkel, Phys. Rev. Lett. 60, 975 (1988).

[4] P. Mazur and W. Van Saarloos, Physica 115A, 21 (1982).

[5] J.A. Somers and P.C. Rem, Shell Conference on Parallel Computing, G.A. van Zee, editor (Lecture Notes on Computer Science 1988).

[6] U. Frisch, D. d'Humières, B. Hasslacher, P. Lallemand, Y. Pomeau and J-P. Rivet, Complex Systems 1, 649 (1987).

[7] M. Henon, Complex Systems 1, 475 (1987).

[8] J-P. Rivet, M. Henon, U. Frisch and D. d'Humières, Europhys. Lett. 7, 231 (1989). 